\title{
Improving the Sustainability of SOA Providers' Networks Via a Collaborative Process Innovation Model
}

\author{
João F. Santanna-Filho ${ }^{1}$, Ricardo J. Rabelo ${ }^{2}$, Peter Bernus ${ }^{3}$, Alexandra A. \\ Pereira-Klen ${ }^{2}$ \\ 1 Federal Rural University of Amazon, Brazil \\ joao.santanna@ufra.edu.br \\ 2 Federal University of Santa Catarina, Florianópolis, Brazil \\ ${ }^{3}$ Griffith University, Australia
}

\begin{abstract}
Companies from the software sector have been seeking new, sustainable business models. A key strategy to achieve is innovation. Being a sector largely formed by SMEs, a general problem is to keep innovation a sustainable practice. Many companies have been shifting their systems' architectures to SOA, but despite its potentials, SOA projects are often costly, complex and risky. One approach to mitigate this is endowing companies with means to innovate collaboratively. Current innovation models are mostly devoted to the manufacturing sector, without supporting the many software and SOA specificities. We present a collaborative innovation process model as a contribution to fill this gap. The model combines open and network innovation approaches and allowing the tailoring of process composition to accommodate the uniqueness of innovation projects. The proposed model was evaluated by industry.
\end{abstract}

Keywords: Collaborative networks · Innovation · Software services

\section{Introduction}

The software sector plays a significant role in the world economy [1]. Advances in Internet technologies and the rise of the services oriented economy challenge the industry to develop new, competitive and sustainable business models [2]. One key strategy to achieve this is innovation [3]. A general problem is that the industry mostly consists of SMEs, which have limited capacity to keep innovation as an ongoing sustainable practice, aligned to their strategies in an environment of permanent change [4].

One of these technological changes is the gradual shift towards implementing systems using the SOA (Service Oriented Architecture) paradigm [5]. In SOA, a software system is formed by a composition of a set of modules - called software services - which are decoupled, distributed, heterogeneous and of several natures, forming a single logical unit to create products and processes [6].

Despite its technological and commercial potentials, SOA projects can be complex and costly, difficult to manage, and hence risky [7]. Existing innovation 
models are basically devoted to manufacturing, without considering the software specificities [8]. This paper exploits the premise that the innovation initiative's risk can be mitigated if the SMEs involved in a given SOA development can jointly innovate, within wider and open networks, sharing risks, investments, resources (human and software/services assets), knowledge and benefits, while keeping their autonomy and independence.

Within this general goal, this paper focuses on the process perspective. It presents the final results of an innovation process model - called SIGMA directed to networks of SOA providers to innovate collaboratively and openly towards generating a valuable outcome in a flexible and non-linear flow considering the particular requirements of every SOA project and its network governance model. Such outcome can be a software prototype, a proof of concepts, a set of ideas for new business models and products, among others. As a value proposition, this model intends to be used as a "guide" that systematizes the innovation process itself. This enables better innovation management, provides a basis for the introduction of good innovation practices, and leverages the continuous maturity improvement of processes, people and the involved organizations.

\section{Basic Concepts and State-of-the-art Reviews}

Collaborative Networks are strategic alliances focused on intense and fluid collaboration among autonomous organizations [9]. In order to support the dynamics of an innovation network, the Virtual Organization (VO) concept is of particular importance. A VO is a temporary and dynamic entity formed out of select autonomous network members that join their complementary core-competencies and general resources to attend to a demand [9].

Innovation Models covering the phases and processes of innovation, via the socalled funnel, namely: selection and/or generation of ideas, concept development, concept evaluation/selection, concept design/specification, implementation and exploitation (adapted from [10]). Innovation models evolved from linear to open and network models [11,12]. Gates are added between steps to constrain the continuation of the process. Processes may be executed in sequence or in parallel, and can go back and forth during the innovation processes. Different types of actors and roles may be involved, including member companies and external supporting partners [13]. A collaborative innovation network is a temporary or long-term alliance of disparate autonomous organizations with the willingness to jointly develop or exploit an innovative business vision by sharing of ideas, knowledge, capacity, resources and services, costs, risks and benefits, supported by the intense use of ICT, and grounded by principles of trust, preparedness, governance and intellectual property rights [8].

A Systematic Literature Review was conducted to oversee the state-of-theart. Journal papers and conference proceedings from 2002 and 2015 were searched in IEE-Explore, ACM, Web of science and ScienceDirect, complemented by the search in the EU CORDIS projects database [14]. The essential search terms used were innovation, collaborative networks, SMEs, SOA \& services. No work 
has been found that dealt with the envisaged open and flexible innovation model based on a network of autonomous SMEs that can participate in all phases of the innovation process, regarding the particular nature of software and SOA. On the other hand, 8 papers $([3,10,11,15,16,17,18,19,20])$ and 7 projects (BIVEE, ComVantage, IMAGINE, CoVES, Laboranova, PLENT and GloNet [[14]]) provided some useful insights for our proposed model. In summary: i) most works are directed to manufacturing, with some of them also covering related services (but not software services); ii) most works presents linear models, where the innovation process seems to follow the same mandatory steps, starting with a business plan, and some aspects that do not fit at all the reality of the software/SOA development process; iii) many models are incomplete in the sense that they support only some phases of innovation; iv) none supports collaboration along the entire innovation process; v) few models cover open innovation; vi) no work gives methodological support to aid companies regarding methods, techniques, etc. recommended to be used in each process.

\section{Proposed Innovation Model}

The model is based on two premises: the innovation process may be serendipitous and unique [21], involves creativity and therefore some unpredictability [22]. Considering this, the model was generally inspired by the Design Thinking method [22] and in particular its notion of innovation "spaces" and evolution stages. This was complemented and adapted taking the six classical innovation processes proposed in [10] into account.

Other requirements are: i) companies may participate in the whole or part of the entire innovation process and related software development cycle, depending on their agreed roles, rights and duties; ii) companies and supporting organizations may join, operate in, and leave the innovation network at different points in time and possibly do so a number of times, during normal network operation or when problems or changes occur; iii) there is no single, predefined or linear progression for the innovation "path".

Our model, called 'SIGMA', supports collaborative innovation from initial exchange of ideas to final delivery/deployment (Fig. 1). The three model spaces represent different moments a typical innovation initiative may go through: (i) Ideas development space: includes processes supporting the management of ideas and their preliminary evaluation/selection. This space is open for all members of the long-term collaborative alliance (e.g. the federation); (ii) Solution development space: includes processes supporting the development of the selected ideas by a VO; i.e. the companies selected considering the required expertise and financial, commercial, technological and software skills; (iii) Solution delivery space: includes processes supporting the delivery of the innovation's outcomes in the case of its commercialization.

The innovation process sequence in these spaces is determined on-the-fly based on the needs of the innovation initiative, i.e. processes may be revisited and some of them (or even an entire space) may not be performed (e.g. the third 


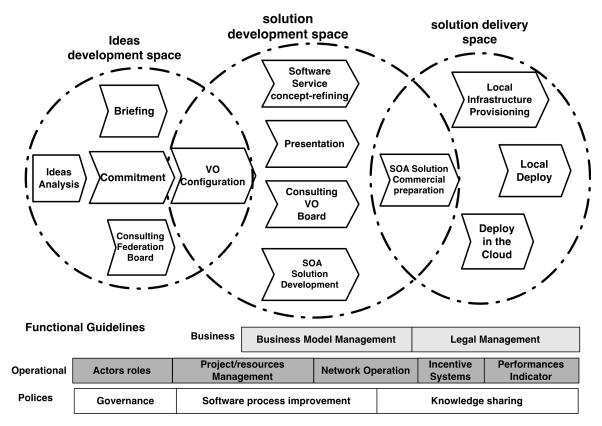

Fig. 1. The SIGMA innovation model

space in case the innovation is not yet ready to be transformed into products). Yet, an initiative does not necessarily have to start from the first space and the ideas analysis process. The innovation can progress in decoupled cycles of evolution between the spaces, where new ideas, prototype versions, goals, etc., can be generated and refined along the time. In order to support such flexibility, and inspired by the SOA paradigm, the model's processes are seen as a set of decoupled building blocks. They act as reference processes that should be selected based on needs and be further instantiated by the VO team.

An innovation initiative can be interrupted, radically changed or shelved (for later use) anytime. The model also uses the classical gate concept for that, although in a slightly different way: a formal evaluation of process outcomes may act as a filter to continue the initiative (decided by the VO) but this is not a mandatory decision.

- Ideas Development Space. Idea Analysis: a company from the alliance can propose a joint innovation to the federation's committee, which will evaluate the initial idea's potential; Briefing: the idea is presented in detail, describing the necessary technologies, potential partnerships, eventual estimated ROI, foreseen market, etc; Commitment: the company that had the original idea may want to protect it using confidentiality contracts before presenting the idea to the other VO members; VO configuration: formation of the VO that will carry out the innovation. This involves partner search and selection, negotiations, setting up VO governance and revenue mode, and contract signature, as well as the definition of performance indicators to be applied upon partners and the innovation process; Consulting federation board: triggered in the case the VO needs advisory about some issues. Although it can depend on how the governance model had been set up, the board usually does not have the power to kill an idea. This board can be formed by some alliance members and may also involve external experts. The main goal here is trying to anticipate problems and propose possible solutions.

- Solution Development Space. Presentation: a more complete project plan and ICT analysis are produced and the eventual business model is 
refined. It also includes issues of IPR and ownership, accounting, and knowledge gaps in the VO and in the federation; Software-service conceptualization: concept development of the SOA solution, the software architecture, the required services to be developed and their functional and non-functional requirements, etc;SOA solution development: it is similar to the previous one, but at a very detailed level. It includes services coding and composition, their integration and final verification. It covers the SOA/services life cycle development $[7,23]$, but respecting the par ticularities when the project is to be performed by a group of companies [24]; Consulting VO Board: process triggered in the case the innovation team needs advisory about some issues, for example, the need for new members; SOA solution commercial preparation: this process ends the SOA solution development space, making the planned outcomes available for whomever has the rights. VO members make agreements and sign contracts, comprising commercial support for the product/service, IPR, commercialization model, price policy, etc.

- Solution Delivery Space. Local infrastructure provisioning: depending on the agreed business model, contracts and QoS requirements, it is necessary to prepare the required infrastructure for the SOA solution deployment at the customer's infrastructure, third party provider, a specific member's site, or the alliance's site; Local deployment: process triggered to make the physical deployment of the SOA solution / product once the infrastructure is ready; Deployment in the cloud: deployment of the SOA solution / product in a third party or alliance's cloud(s).

- Functional Guidelines (FG). Network members are basically SMEs, and their managers may not be fully aware of the issues to consider in each of the processes. FGs correspond to supporting methods, techniques and tools that may be considered by the VO team during collaborative innovation. Ten FGs have been identified after a compilation of several papers on innovation (e.g. $[25,26])$. FGs are grouped into business, operational and policies levels:( i) Business level: FGs related to commercialization of innovation. Involves the issues of Business Model management and Legal aspects management; (ii) Operational level: FGs to support the daily operation of the innovation development. Involves the issues of Actors' roles, Project/Resources management, VO Network operation, Incentive systems, and Performance indicators; (iii) Policies level: FGs related to relations within the VO, the VO with other actors (internal or external), and with customers. Involves the issues of Governance, Software process improvement, and Knowledge sharing and management. The proposed model does not offer such methods, tools, etc.. Instead, adopting the vision of reference models, they have to be instantiated regarding existing practices, available financial resources, prepared people, etc.

\section{Model Validation}

The SIGMA model was evaluated by a group of SOA experts from a cluster of ICT/SOA provider companies located in two cities in the South of Brazil. A total 
of 29 professionals from 17 companies were involved in three small workshops. The selected companies had some level of experience on innovation.

The model was presented and doubts clarified. Hypothetical but representative business cases were proposed by them so as to simulate near-real innovation scenarios. The professionals were split into groups in order to also simulate autonomous companies. The authors of this research acted as "moderators", sometimes representing members of the Network or the VO Board.

A survey composed of 18 questions was prepared adopting the GQM method [27] and applied to the companies after the group dynamics. Twelve objective questions were answered using the Likert scale and there were 6 open questions.

In summary: i) $90 \%$ agreed about the separation of the process model into 3 spaces; ii) $80 \%$ fully agreed that the process systematization has the potential to decrease costs, time and/or risks of SOA innovation projects; iii) $100 \%$ agreed that the model properly supports the required flexibility of innovation in software and SOA; iv) all devised processes and FGs were considered as pertinent, although with different levels of importance; v) $100 \%$ agreed that SMEs of software providers will need to adopt more formal innovation practices and will be involved in wider value chains in the next 5 years; vi) although more directed to SMEs, $50 \%$ pointed out a sort of technical and cultural obstacles as well as organization impacts to adopt the model.

Some initiatives were mentioned by the companies as enablers for the model adoption, like the creation of common training courses on the several related issues; the strengthening of the collaboration culture and trust among companies; and adequate legal frameworks also considering intellectual property rights. On the other hand, some barriers to adopt the model were also identified, like the lack of governance culture at the network level; the lack of systematic collaboration culture by SMEs; and lack of ICT tools to support the distributed innovation and project/innovation management.

\section{Conclusions}

This paper presented the final results of a novel innovation process model devoted to support collaborative innovation among software services provider SMEs. The model has been developed with the view of Collaborative Networks providing the foundation for supporting SMEs to work as a network, sharing costs, risks and benefits, where an innovation initiative is operated as a Virtual Organization (VO).

The most relevant supporting constituents to be taken into account in the innovation process were identified and briefly described. They are called 'functional guidelines' and aim at helping companies to be aware of the issues to be managed in the collaborative innovation life cycle.

The model presents a number of new contributions compared to related works, namely: it is directed to software and SOA; it introduces the concept of 'functional guidelines'; the model allows flexibility, non-linearity and on-the-fly 
composability of the innovation process for every innovation initiative; it introduces the concept of 'building block' as a reference process to be instantiated according to existing cultural or other general conditions; it adapts the SOA development process to cope with the requirements of collaborative innovation among disparate companies.

From the business standpoint, the adoption of the proposed model has the potential to provide a number of benefits, such as: the sharing of risks among partners, which decreases their losses in the case if unsuccessful innovation initiatives; faster response to innovation demands; greater synergy and networking among companies so generating a wider scope of complementary skills that can be used in future businesses in other value chains; higher ROI as companies' services can be reused by other companies; newer business models derived from the networked business architecture; and the breeding of more intense culture of innovation in the companies, reinforcing the ecosystem's strength.

Working collaboratively is a learning process. Companies are heterogeneous (culture, working methods, human resources' qualification, core competences, etc.) and autonomous, meaning that their different strategies must be accommodated and interoperate regarding their different priorities, exploitation plans, and trade-offs in terms of acceptable risks, trust and benefits. Although presented in its final version, the model cannot be seen as fixed. New processes and FGs can be added and existing one may evolve.

The innovation process model is not an end in itself. It is part of a much wider framework instead. Complementary issues, like having an innovation model, fostering and reinforcing leadership and innovation culture, having appropriate legal and funding mechanisms, among many other conditions, should be developed and supported for a successful and healthy innovation environment. The next main step of this research refers to validate the model in real cases and to test it close to a greater number of companies.

\section{References}

1. Kramer, W.J., Jenkins, B., Katz, R.S.: The Role of the Information and Communications Technology Sector in Expanding Economic Opportunity. Harvard University, Cambridge, MA (2007)

2. Nie, Z.: Credibility Evaluation of Saas Tenants. In: International Conference on Advanced Computer Theory and Engineering. vol. 4, pp. 488-491. IEEE (2010)

3. Li, Y., Shen, J., Shi, J., Shen, W., Huang, Y., Xu, Y.: Multi-model Driven Collaborative Development Platform for Service-oriented E-Business Systems. Advanced Engineering Informatics 22, 328-339 (2008)

4. Westphal, I., K.D., Thoben, Seifert, M.: Managing Collaboration Performance to Govern Virtual Organizations. J. of Int. Manufacturing 21(3), 311-320 (2010)

5. PRWEB: http://www.prweb.com

6. Papazoglou, M.: Web Services: Principles and Technology. Pearson Edu. (2008)

7. O'Brien, L., Merson, P., Bass, L.: Quality Attributes for Service-oriented Architectures. In: Proceedings of the International Workshop on Systems Development in SOA Environments. p. 3. IEEE (2007) 
8. Santanna-Filho, J.F., Rabelo, R.J., Pereira-Klen, A.A.: An Innovation Model for Collaborative Networks of SOA-Based Software Providers, pp. 169-181. Springer Berlin Heidelberg, Berlin, Heidelberg (2014)

9. Afsarmanesh, H., Camarinha-Matos, L., Ollus, M.: Methods and Tools for Collaborative Networked Organizations. Springer Science Business Media (2008)

10. du Preez, N.D., Louw, L.: A Framework for Managing the Innovation Process. In: PICMET '08 - 2008 Portland International Conference on Management of Engineering Technology. pp. 546-558 (July 2008)

11. Rycroft, R., Kash, D.: Self-organizing Innovation Networks: Implications for Globalization. Technovation 24, 187-197 (2010)

12. Chesbrough, H.W.: Open innovation: The new imperative for creating and profiting from technology. Harvard Business Press (2006)

13. Tidd, J., Bessant, J., Pavitt, K.: Innovation Management. Wiley (2001)

14. CORDIS: http://cordis.europa.eu/projects/home_en.html

15. Janner, T., Schroth, C., Schmid, B.: Modelling Service Systems for Collaborative Innovation in the Enterprise Software Industry-the st. Gallen Media Reference Model Applied. In: IEEE SCC. vol. 2, pp. 145-152. IEEE (2008)

16. Berre, A.J., Lew, Y., Elvesæter, B., de Man, H.: Service Innovation and Service Realisation with VDML and ServiceML. In: 17th IEEE International Enterprise Distributed Object Computing Conference Workshops. pp. 104-113. IEEE (2013)

17. Kourtesis, D., Bratanis, K., Bibikas, D., Paraskakis, I.: Software Co-development in the era of Cloud Application Platforms and Ecosystems: the Case of CAST. In: Working Conference on Virtual Enterprises. pp. 196-204. Springer (2012)

18. Hoyer, V., Christ, O.: Collaborative E-business Process Modelling: A Holistic Analysis Framework Focused on Small and Medium-sized Enterprises. In: International Conference on Business Information Systems. pp. 41-53. Springer (2007)

19. Belussi, F., Arcangeli, F.: A Typology of Networks: Flexible and Evolutionary Firms. Research policy 27(4), 415-428 (1998)

20. Camarinha-Matos, L.M., Afsarmanesh, H., Oliveira, A.I., Ferrada, F.: Cloud-based Collaborative Business Services Provision. In: International Conference on Enterprise Information Systems. pp. 366-384. Springer (2013)

21. Hwang, V.W., Horowitt, G.: The Rainforest: The Secret to Building the Next Silicon Valley. Regenwald Los Altos Hills, CA (2012)

22. Mootee, I.: Design Thinking for Strategic Innovation: What They Can't Tteach you at Business or Design School. John Wiley \& Sons (2013)

23. Kontogiannis, K., Lewis, G.A., Smith, D.B.: A research agenda for service-oriented architecture. In: Proceedings of the 2nd international workshop on Systems development in SOA environments. pp. 1-6. ACM (2008)

24. Cancian, M.H., Rabelo, R.J., von Wangenheim, C.G.: Supporting Processes for Collaborative SaaS. In: Working Conference on Virtual Enterprises. pp. 183-190. Springer (2013)

25. Munkongsujarit, S., Srivannaboon, S.: Key Success Factors for Open Innovation Intermediaries for SMEs: A Case Study of ITAP in Thailand. In: 2011 Proceedings of PICMET'11. pp. 1-8. IEEE (2011)

26. Van Zyl, J.: Process Innovation Imperative [Software Product Development Organisation]. In: Change Management and the New Industrial Revolution, 2001. IEMC'01 Proceedings. pp. 454-459. IEEE (2001)

27. Basili, V.R., Caldiera, G., Rombach, H.D.: The Goal Question Metric Approach. In: Encyclopedia of Software Engineering. Wiley (1994) 